\title{
The structure of mode-locked regions in quasi-periodically forced circle maps
}

\author{
P. Glendinning ${ }^{\mathrm{a}, *}$, U. Feudel $^{\mathrm{b}}$, A.S. Pikovsky ${ }^{\mathrm{b}}$, J. Stark $^{\mathrm{c}}$ \\ a Department of Mathematics, UMIST, P.O. Box 88, Manchester M60 1QD, UK \\ ${ }^{\mathrm{b}}$ Institute for Theoretical Physics and Astrophysics, University of Potsdam, Am Neuen Palais, PF 601553, D-14415 Potsdam, Germany \\ ${ }^{c}$ Centre for Dynamical Systems and its Applications, Civil Engineering Building, University College London, Gower Street, \\ London WC1E 6BT, UK
}

Received 3 February 1999; received in revised form 23 November 1999; accepted 26 November 1999

Communicated by J.D. Meiss

\begin{abstract}
Using a mixture of analytic and numerical techniques we show that the mode-locked regions of quasi-periodically forced Arnold circle maps form complicated sets in parameter space. These sets are characterized by 'pinched-off' regions, where the width of the mode-locked region becomes very small. By considering general quasi-periodically forced circle maps we show that this pinching occurs in a broad class of such maps having a simple symmetry. (C 2000 Elsevier Science B.V. All rights reserved.
\end{abstract}

PACS: 05.45.+b

Keywords: Mode-locked region; Quasi-periodically forced circle maps

\section{Introduction}

The Arnold circle map

$$
x_{n+1}=x_{n}+\Omega+\frac{A}{2 \pi} \sin 2 \pi x_{n}, \quad 0 \leq A<1, \quad 0 \leq \Omega<1,
$$

has become a paradigm for the study of a wide variety of periodically forced systems in physics and biology. The dynamics of this map may be characterized by the rotation number

$$
\rho(\Omega, A)=\lim _{n \rightarrow \infty} \frac{x_{n}-x_{0}}{n},
$$

\footnotetext{
* Corresponding author.
}

0167-2789/00/\$ - see front matter @ 2000 Elsevier Science B.V. All rights reserved. PII: S 0 167-2789(99)00235-3 
which is known to exist and is independent of $x_{0}$. Intuitively, the rotation number measures the average angular velocity of an orbit around the circle $\{x(\bmod 1)\}$. If $\rho \in \mathbb{Q}$ then the Arnold map (projected onto the circle) has periodic points, whilst if $\rho \neq \mathbb{Q}$ then there are no periodic points on the circle. It is natural to describe the parameter plane $(\Omega, A)$ by rotation number, defining regions

$$
\mathcal{L}_{\omega}=\{(\Omega, A) \mid \rho(\Omega, A)=\omega\} .
$$

If $\omega$ is rational, $\omega=p / q$ say, then $\mathcal{L}_{p / q}$ is a 'tongue' with non-empty interior emanating from $(\Omega, A)=(p / q, 0)$ into $A>0$. These tongues are referred to as mode-locked regions. On the boundary of a tongue, two or more periodic orbits annihilate in saddle-node bifurcations. On the other hand, if $\omega \notin \mathbb{Q}$ then $\mathcal{L}_{\omega}$ is generally assumed to be a curve stretching from $(\omega, 0)$ to $\left(\omega^{\prime}, 1\right)$ for some $\omega^{\prime} \in \mathbb{R}$. This characteristic picture gives insight (for example) into the devil's staircase of rotation numbers obtained in a number of real systems: the plateaus of the staircase correspond to parameter values in the mode-locked tongues (see e.g. [2]).

Recently, there has been an explosion of interest in quasi-periodically forced systems (systems forced at two independent frequencies). The natural extension of (1.1) to this situation is the quasi-periodically forced circle map

$$
x_{n+1}=x_{n}+\Omega+\frac{A}{2 \pi} \sin 2 \pi x_{n}+B \sin 2 \pi \theta_{n}, \quad \theta_{n+1}=\theta_{n}+\alpha,
$$

where $\alpha \in(0,1)$ is irrational, $0 \leq A, \Omega<1$ and $B \geq 0$. The restriction on $A$ ensures that (1.4) is a diffeomorphism (i.e., smooth with smooth inverse). The new variable $\theta$ represents the phase of the second forcing frequency, and $B$ is a coupling parameter. Such systems have attracted attention both because of their relevance as paradigms for physical situations and because strange non-chaotic attractors may be found numerically at some values of the parameters (e.g. $[9,10,12,13,15,17])$. As with the Arnold circle map (1.1), it is natural to define rotation numbers in the $\theta$-direction and the $x$-direction. Clearly

$$
\lim _{n \rightarrow \infty} \frac{\theta_{n}-\theta_{0}}{n}=\alpha
$$

but the existence of

$$
\rho(\alpha, \Omega, A, B)=\lim _{n \rightarrow \infty} \frac{x_{n}-x_{0}}{n}
$$

is not obvious. However, in a much more general context, Herman [11] has shown that this rotation number does indeed exist and is independent of $\left(x_{0}, \theta_{0}\right)$ provided, as will be assumed throughout this paper, $\alpha \notin \mathbb{Q}$. Henceforth the arguments of $\rho$ will be taken as understood, so $\rho$ will denote (1.5), and not the rotation number of the circle $\operatorname{map}(1.2)$.

Since $\alpha$ is irrational (1.4) has no periodic orbits. However, there is still a natural dichotomy of the dynamics. For the quasi-periodically forced circle map mode-locking may occur if the rotation number $\rho$ is rationally related to either of the forcing frequencies (viz. 1 and $\alpha$ ) or to a combination of these. Thus, the dynamics of (1.4) is said to be mode-locked if $\rho \in \alpha \mathbb{Q}+\mathbb{Q}$, i.e., if there exist rational numbers $r_{1}$ and $r_{2}$ such that $\rho=r_{1} \alpha+r_{2}$. If no such relationship holds then the dynamics is said to be incommensurate. There is a standard dynamics associated with each of these two possibilities: if the dynamics is mode-locked then the attractor may be a finite union of closed invariant curves on the torus $(x(\bmod 1), \theta(\bmod 1))$, whilst if the dynamics is incommensurate then the attractor may be the whole torus, with all orbits being dense on the torus. In both cases there are conjectural non-standard dynamics which may occur at some parameter values, and which would explain the appearance of strange non-chaotic attractors $[5,7,8]$.

By analogy with the circle map (1.1), a natural step in the analysis of (1.4) is to determine the nature of the mode-locked regions in parameter space 


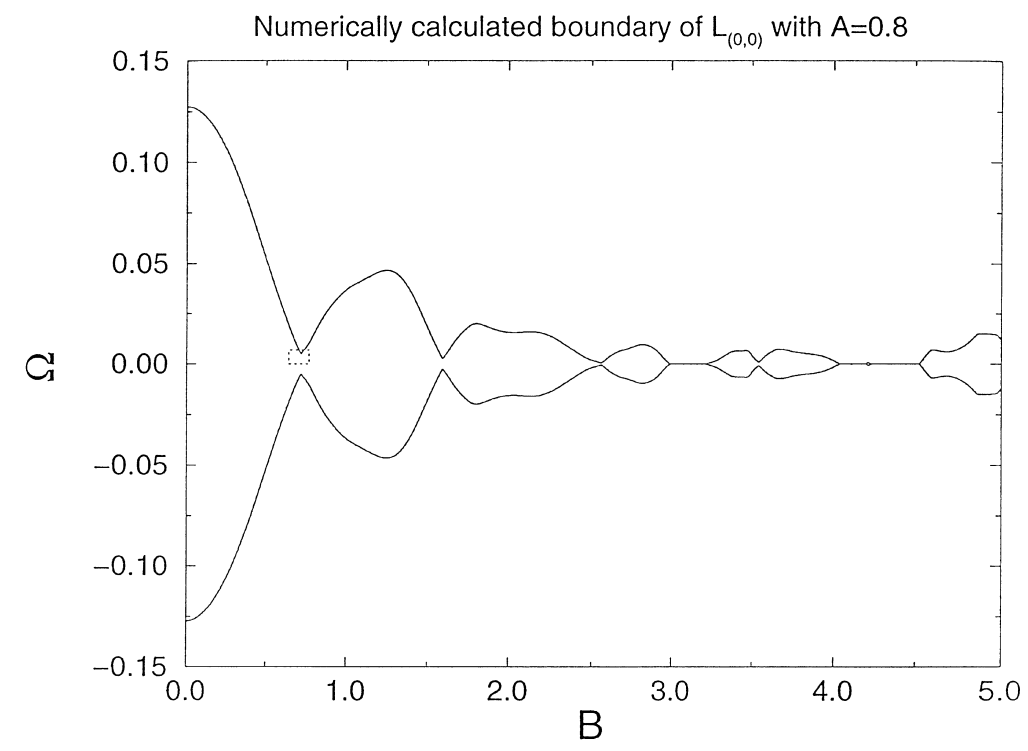

Fig. 1. Numerically calculated boundary of $\mathcal{L}_{(0,0)}$ for (1.4) with $\alpha=\frac{1}{2}(\sqrt{5}-1)$ and $A=0.8$.

$$
\mathcal{L}_{r_{1}, r_{2}}=\left\{(\alpha, \Omega, A, B) \mid \rho=r_{1} \alpha+r_{2}, \quad r_{i} \in \mathbb{Q}, i=1,2\right\}
$$

Although partial (numerical) descriptions have been given [4-6,16] we know of no systematic study of these regions along the lines of the standard analysis for circle maps. The aim of this paper is to provide some details of these regions. In particular, asymptotic analysis for small $A$ is used to describe $\mathcal{L}_{(0,0)}$ and $\mathcal{L}_{(1,0)}$ in some detail, and numerical experiments are used to show how the pictures obtained are modified outside the region of validity of this analysis. Some higher order mode-locked tongues are also amenable to a partial analysis, and these are included for completeness. As Ding et al. [5] suggest, the width of the mode-locked regions tends to zero at large $B$, and we are able to show that the decay of this width is proportional to $B^{-1 / 2}$ for moderate values of $B$. More striking is the fact that this decay is modulated and there are values of $B$ at which the width of the regions goes to zero at lowest order, although higher order terms which have not been calculated may imply that the tongues have a very small, but finite, width at such values. This 'pinching' of mode-locked tongues has been observed numerically [6,7] but the results presented below suggest that it is a persistent feature of these systems. Fig. 1 shows the boundary of $\mathcal{L}_{(0,0)}$ in the $(\Omega, B)$ plane computed numerically for fixed $\alpha=\frac{1}{2}(\sqrt{5}-1)$ and $A=0.8$ (cf. [7]). The tongue appears to open and close as $B$ is increased. At smaller values of $A$, as will be shown later, this oscillation is more regular. Moreover, the oscillations in the boundary clearly decrease in amplitude as $B$ increases; the scaling referred to above will be investigated in later figures.

The remainder of this paper is organized as follows. In Sections $2-4$ we present the perturbation analysis for $\mathcal{L}_{(0,0)}, \mathcal{L}_{(1,0)}$ and some higher order tongues, together with numerical confirmation of the results. In Section 5, we describe the results of numerical experiments at larger values of $A$ and comment on the similarities and differences observed. We also look more closely at the 'pinching' effect at small $A$ and show that higher order mode-locked regions display similar effects. In Section 6, we use the method of stationary phase to argue that the description obtained should be qualitatively independent of the precise details of the quasi-periodically forced circle map being discussed provided the map retains a simple symmetry. Appendix A gives the standard, but nasty, integrals which need to be evaluated during the course of the analysis. 


\section{The mode-locked region $\mathcal{L}_{(0,0)}(A$ small $)$}

The calculation for $\mathcal{L}_{(0,0)}$ will be given in some detail; since the basic methods are the same this level of detail will not be provided in subsequent sections. Throughout $\alpha$ is assumed to be a fixed irrational number, $0<\alpha<1$. The approach is similar to that of Heagy and Hammel [10] for the quasi-periodically forced logistic map: we look for invariant curves of a particular form and solve the lowest order perturbation problem to obtain a solution involving an arbitrary parameter. This parameter is then fixed by a solvability condition at the next order - a condition which can only be satisfied provided $(\Omega, A, B)$ lies in a restricted region of parameter space. This region will be the lowest order approximation to the mode-locked region. It is worth emphasising that the analysis presented below is only valid for $A$ small and $B$ of order 1 compared to $A$. Although, we will talk of 'large $B$ ' this must be understood as $B$ large, but small compared to $A^{-1}$ !

Let $A \sim a \epsilon$ and $\Omega \sim \omega \epsilon$ for some small parameter $\epsilon, 0<\epsilon \ll 1$, with $a, \omega \geq 0$. The quasi-periodically forced circle map (1.4) is thus

$$
x_{n+1} \sim x_{n}+B \sin 2 \pi \theta_{n}+\epsilon\left(\omega+\frac{a}{2 \pi} \sin 2 \pi x_{n}\right), \quad \theta_{n+1}=\theta_{n}+\alpha .
$$

If $\rho=0$ then it is natural to look for invariant curves

$$
x=G(\theta), \quad G(\theta+1)=G(\theta),
$$

which can be expanded as an asymptotic series in $\epsilon$,

$$
G(\theta) \sim G_{0}(\theta)+\epsilon G_{1}(\theta), \quad G_{i}(\theta+1)=G_{i}(\theta), \quad i=0,1 .
$$

Substituting this ansatz into (2.1) and isolating terms of order $\epsilon^{0}$ and $\epsilon^{1}$, respectively, yields two equations for $G_{0}$ and $G_{1}$,

$$
G_{0}(\theta+\alpha)=G_{0}(\theta)+B \sin 2 \pi \theta,
$$

and

$$
G_{1}(\theta+\alpha)=G_{1}(\theta)+\omega+\frac{a}{2 \pi} \sin 2 \pi G_{0}(\theta) .
$$

The lowest order problem, then (2.4), may be solved by posing $G_{0}(\theta)=\sum g_{k} \exp (2 \pi \mathrm{i} k \theta)$ to give

$$
\begin{aligned}
G_{0}(\theta) & =g_{0}+\frac{B}{2(1-\cos 2 \pi \alpha)}(\sin 2 \pi(\theta-\alpha)-\sin 2 \pi \theta)=g_{0}-\frac{B}{2 \sin \pi \alpha} \cos 2 \pi\left(\theta-\frac{1}{2} \alpha\right) \\
& =g_{0}-\frac{B}{2 \sin \pi \alpha} \sin 2 \pi(\theta-\phi),
\end{aligned}
$$

where $g_{0}$ is an arbitrary constant and $\phi=\frac{1}{2} \alpha-\frac{1}{4}$. The extra phase shift in the last line of (2.6) is trivial, but it means that the integrals evaluated below are in a standard form. We now return to the second perturbation equation (2.5). To obtain a consistency condition which fixes the value of $g_{0}$, integrate (2.5) from 0 to 1 in $\theta$; $\int_{0}^{1} G_{1}(\theta+\alpha) \mathrm{d} \theta=\int_{0}^{1} G_{1}(\theta) \mathrm{d} \theta$ as $G_{1}(\theta+1)=G_{1}(\theta)$, so this gives

$$
0=\omega+\frac{a}{2 \pi} \int_{0}^{1} \sin 2 \pi G_{0}(\theta) \mathrm{d} \theta .
$$

Substituting (2.6) into (2.7) and using (A.7) from Appendix A this implies that

$$
-\frac{2 \pi \omega}{a}=\sin 2 \pi g_{0} J_{0}\left(\frac{\pi B}{\sin \pi \alpha}\right)
$$




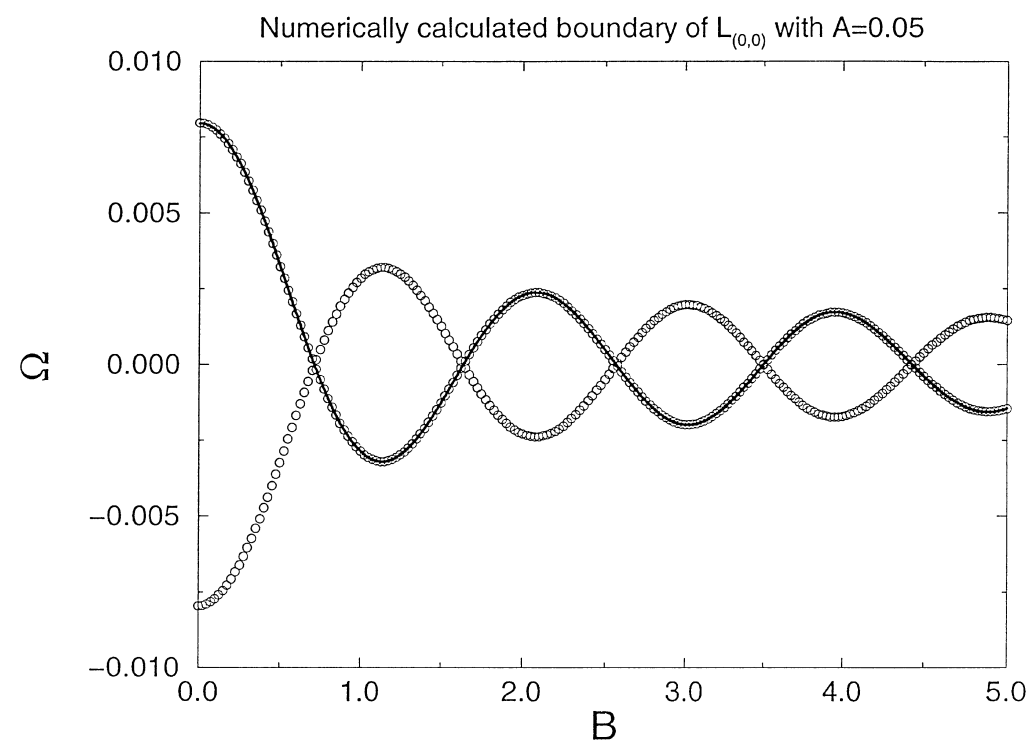

Fig. 2. The boundary of $\mathcal{L}_{(0,0)}$ for (1.4) with $\alpha=\frac{1}{2}(\sqrt{5}-1)$ and $A=0.05$ (numerically computed data points are shown as circles) together with the prediction, (2.10) which is shown as a continuous curve.

where $J_{0}(z)$ is the Bessel function of order zero. From (2.8) it is immediate that the constant $g_{0}$ can be determined provided

$$
\left|J_{0}\left(\frac{\pi B}{\sin \pi \alpha}\right)\right| \geq \frac{2 \pi \omega}{a} \sim \frac{2 \pi \Omega}{A} .
$$

This set defines the approximate phase-locked region $\mathcal{L}_{(0,0)}$ for $A$ sufficiently small. In particular, the boundary of this region is

$$
\Omega \sim \frac{A}{2 \pi}\left|J_{0}\left(\frac{\pi B}{\sin \pi \alpha}\right)\right|
$$

The numerically computed boundary, together with this theoretical prediction, is illustrated in Fig. 2. The agreement between theory and experiment is excellent for $A=0.05$. Note that $J_{0}(0)=1$, so that as $B \rightarrow 0(2.9)$ tends to the standard result for the Arnold circle map as expected.

At large $B$ (2.10) becomes (using (A.8))

$$
\Omega \sim \frac{A}{2 \pi^{2}}\left(\frac{2|\sin \pi \alpha|}{B}\right)^{1 / 2}\left|\cos \left(\frac{\pi B}{|\sin \pi \alpha|}-\frac{\pi}{4}\right)\right|,
$$

showing that the decay in the envelope of the boundary is proportional to $B^{-1 / 2}$. Ding et al. [5] predict that the tongue would get small at large $B$, but do not suggest any scaling. Moreover, from the oscillating cosine term in (2.11) we see that the width of the tongues is zero to lowest order at zeroes of the Bessel function, i.e., at

$$
B_{m} \sim\left(m-\frac{1}{4}\right)|\sin \pi \alpha| .
$$

This pinching effect for the phase-locked regions will recur throughout this paper. 


\section{The mode-locked region $\mathcal{L}_{(1,0)}(A$ small $)$}

In the limit of $B \rightarrow 0$ (the Arnold circle map), $\mathcal{L}_{(1,0)}$ is qualitatively different from $\mathcal{L}_{(0,0)}$; it is a curve in the $(\Omega, A)$ plane rather than a tongue. We therefore expect the width of $\mathcal{L}_{(1,0)}$ tends to zero as $B \rightarrow 0$. To obtain an asymptotic formula for such a region for small $A$ the parameters must be rescaled as $A \sim a \epsilon$ and $\Omega \sim \alpha+\omega \epsilon$ $(0<\epsilon \ll 1)$ so that $(1.4)$ becomes

$$
x_{n+1} \sim x_{n}+\alpha+B \sin 2 \pi \theta_{n}+\epsilon\left(\omega+\frac{a}{2 \pi} \sin 2 \pi x_{n}\right), \quad \theta_{n+1}=\theta_{n}+\alpha .
$$

From considerations of the homotopy type, invariant curves in $\mathcal{L}_{(1,0)}$ will be of the form

$$
x=G(\theta), \quad G(\theta+1)=G(\theta)+1,
$$

and so we pose an asymptotic solution of the form

$$
x \sim G_{0}(\theta)+\epsilon G_{1}(\theta),
$$

with $G_{0}(\theta+1)=G_{0}(\theta)+1$ and $G_{1}(\theta+1)=G_{1}(\theta)$. Substituting into (3.1) and equating terms of order $\epsilon^{0}$ and $\epsilon^{1}$, respectively, gives

$$
G_{0}(\theta+\alpha)=G_{0}(\theta)+\alpha+B \sin 2 \pi \theta
$$

and

$$
G_{1}(\theta+\alpha)=G_{1}(\theta)+\omega+\frac{a}{2 \pi} \sin 2 \pi G_{0}(\theta) .
$$

If we pose $G_{0}(\theta)=\theta+h(\theta)$ with $h(\theta+1)=h(\theta)$ then $h(\theta)$ satisfies (2.4) and so, using (2.6),

$$
G_{0}(\theta)=g_{0}+\theta-\frac{B}{2 \sin \pi \alpha} \sin 2 \pi(\theta-\phi),
$$

where $g_{0}$ is an arbitrary constant which we hope to determine at next order and $\phi=\frac{1}{2} \alpha-\frac{1}{4}$. The consistency condition obtained from integrating (3.5) between 0 and 1 is

$$
0=\omega+\frac{a}{2 \pi} \int_{0}^{1} \sin 2 \pi\left(g_{0}+\theta-\frac{B}{2 \sin \pi \alpha} \sin 2 \pi(\theta-\phi)\right) \mathrm{d} \theta
$$

which can be rewritten, using (A.7) in Appendix A, as

$$
0=\omega-\frac{a}{2 \pi} \sin 2 \pi\left(g_{0}+\phi\right) J_{1}\left(\frac{\pi B}{\sin \pi \alpha}\right),
$$

where $J_{1}(z)$ is the Bessel function of order 1. Thus, as in Section 2, we find that (3.8) can be solved for $g_{0}$ provided

$$
\left|J_{1}\left(\frac{\pi B}{\sin \pi \alpha}\right)\right| \geq \frac{2 \pi|\omega|}{a} \sim \frac{2 \pi|\Omega-\alpha|}{A},
$$

and so for sufficiently small $A$ the boundary of the mode-locked region $\mathcal{L}_{(1,0)}$ is given to lowest order by

$$
|\Omega-\alpha| \sim \frac{A}{2 \pi}\left|J_{1}\left(\frac{\pi B}{|\sin \pi \alpha|}\right)\right| .
$$

Fig. 3 shows a comparison between this prediction and numerically calculated points on the boundary of $\mathcal{L}_{(1,0)}$ for $A=0.05$. Once again the agreement is excellent and the boundary shows the $B^{-1 / 2}$ narrowing and the regular 


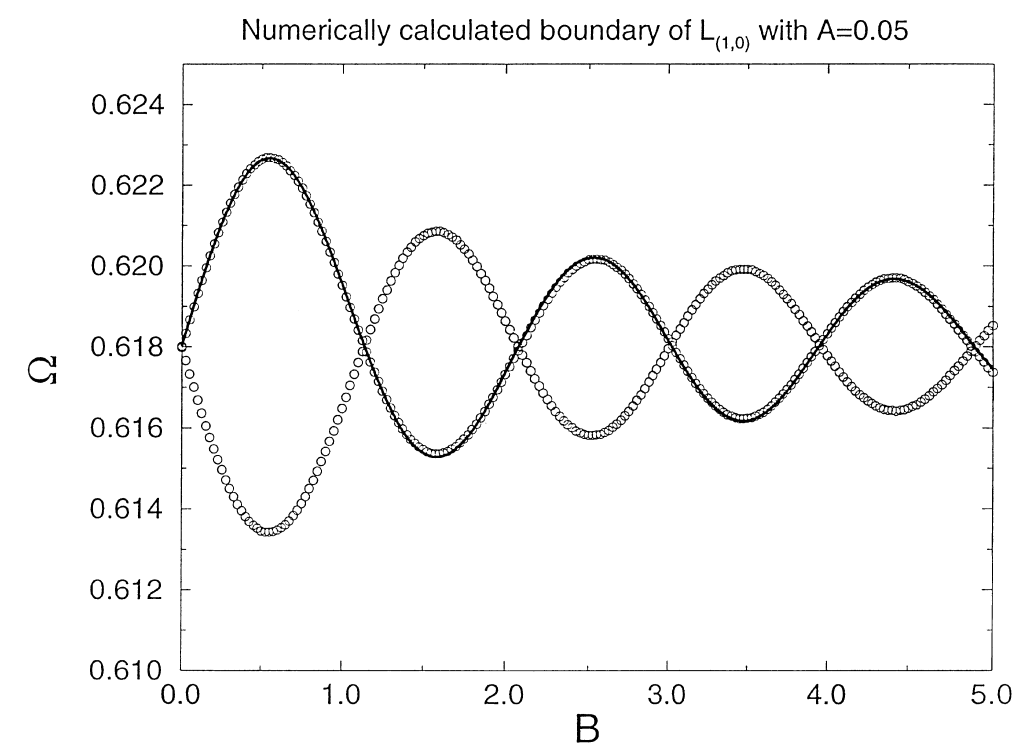

Fig. 3. The boundary of $\mathcal{L}_{(1,0)}$ for (1.4) with $\alpha=\frac{1}{2}(\sqrt{5}-1)$ and $A=0.05$ computed numerically (data points are indicated by circles) together with the prediction, (3.10), which is shown as a continuous curve.

pinching effect as expected from the large $z$ asymptotics of $J_{1}(z)$ (see Appendix A, (A.8)) from which we obtain, for large $B$ and very small $A$,

$$
|\Omega-\alpha| \sim \frac{A}{2 \pi^{2}}\left(\frac{2|\sin \pi \alpha|}{B}\right)^{1 / 2}\left|\cos \left(\frac{\pi B}{|\sin \pi \alpha|}-\frac{3}{4} \pi\right)\right| .
$$

Moreover, since $J_{1}(0)=0$, the tongue closes as $B \rightarrow 0$ as expected from the comments about the tongue structure for the Arnold circle map made at the beginning of this section.

\section{Higher order mode-locked regions ( $A$ small)}

For a general mode-locked region with $\rho=(p / q) \alpha+(r / s)$ the most simple solutions will lie on the union of $s$ closed curves which wrap $q$ times around the torus and are shifted by $r$ in the $x$-direction after $s$ iterations of the map. Thus, these curves are of the form

$$
\frac{p}{q} \theta+G(\theta), \quad G(\theta+q)=G(\theta)
$$

In principle (4.1) could be used to generate first-order conditions as in previous sections, but the computations rapidly become extremely complicated and we shall restrict ourselves to cases which illustrate the possibilities for higher order mode-locked regions. These are $\mathcal{L}_{\left(r_{1}, r_{2}\right)}$ with $\left(r_{1}, r_{2}\right)$ equal to $\left(0, \frac{1}{2}\right)$ and $(p / q, 0)$.

To determine $\mathcal{L}_{(0,1 / 2)}$ we will need the second iterate of the map to lowest order, so setting $\left(r_{1}, r_{2}\right)=\left(0, \frac{1}{2}\right)$ we pose $A \sim a \epsilon$ and $\Omega \sim r_{1} \alpha+r_{2}+\omega \epsilon$ to obtain

$$
\begin{aligned}
x_{n+2} & \sim x_{n}+2\left(r_{1} \alpha+r_{2}\right)+B \sin 2 \pi \theta_{n}+B \sin 2 \pi \theta_{n+1} \\
& +\epsilon\left(2 \omega+\frac{a}{2 \pi} \sin 2 \pi x_{n}+\frac{a}{2 \pi} \sin 2 \pi\left(x_{n}+r_{1} \alpha+r_{2}+B \sin 2 \pi \theta_{n}\right)\right),
\end{aligned}
$$

with $\theta_{n+1}=\theta_{n}+\alpha$ as usual. 
To obtain the lowest order approximation to $\mathcal{L}_{(0,1 / 2)}$ we pose the asymptotic solution $x \sim G_{0}(\theta)+\epsilon G_{1}(\theta)$, where $G_{i}(\theta+1)=G_{i}(\theta), i=0,1$. Substituting into (4.2) and isolating terms of order $\epsilon^{0}$ and $\epsilon^{1}$, respectively, (noting that we expect the second iterate of the invariant curve to be the invariant curve shifted by 1 in the $x$-direction) gives

$$
G_{0}(\theta+2 \alpha)=G_{0}(\theta)+B \sin 2 \pi \theta+B \sin 2 \pi(\theta+\alpha)
$$

and

$$
G_{1}(\theta+2 \alpha)=G_{1}(\theta)+2 \omega+\frac{a}{2 \pi} \sin 2 \pi G_{0}(\theta)+\frac{a}{2 \pi} \sin 2 \pi\left(G_{0}(\theta)+\frac{1}{2}+B \sin 2 \pi \theta\right) .
$$

The leading order equation is easily solved, so the consistency condition is

$$
0=2 \omega+\frac{a}{2 \pi} \int_{0}^{1}\left(\sin 2 \pi G_{0}(\theta)-\sin 2 \pi\left(G_{0}(\theta)+B \sin 2 \pi \theta\right)\right) d \theta
$$

Although messy, the integral can be evaluated and turns out to be identically equal to zero (we omit the boring calculation which leads to this boring conclusion). Hence $\omega=0$ and we find essentially the same conclusion as for the circle map:

$$
|2 \Omega-1| \sim \mathrm{O}\left(A^{2}\right) .
$$

After this rather disappointing result we move on to the general tongue $\mathcal{L}_{(p / q, 0)}$. Since $r_{2}=0$ the solution curve is invariant, i.e., it is not necessary to consider the second iterate of the map. Posing $A \sim a \epsilon, \Omega \sim(p / q) \alpha+\omega \epsilon$ and an invariant curve of the form $(p / q) \theta+G_{0}(\theta)+\epsilon G_{1}(\theta)$ with $G_{i}(\theta+q)=G_{i}(\theta), i=0$, we obtain from (2.1) that

$$
G_{0}(\theta+\alpha)=G_{0}(\theta)+B \sin 2 \pi \theta
$$

and

$$
G_{1}(\theta+\alpha)=G_{1}(\theta)+\omega+\frac{a}{2 \pi} \sin 2 \pi\left(\frac{p}{q} \theta+G_{0}(\theta)\right) .
$$

These equations are very similar to those obtained in Section 3, the only subtlety is that the period of $G_{i}$ is now $q$ rather than 1. By the same methods as used in earlier sections, (4.7) may be solved to obtain (as before)

$$
G_{0}(\theta)=g_{0}-\frac{B}{2 \sin \pi \alpha} \sin 2 \pi(\theta-\phi)
$$

and so the first-order consistency condition, obtained by integrating (4.8) from 0 to $q$, is

$$
0=q \omega+\frac{a}{2 \pi} \int_{0}^{q} \sin 2 \pi\left(\frac{p}{q} \theta+g_{0}-k \sin 2 \pi(\theta-\phi)\right) \mathrm{d} \theta,
$$

where $k=B / 2 \sin \pi \alpha$. Let $g_{0}^{\prime}=g_{0}+(p \phi / q)$ then (4.10) is

$$
\begin{aligned}
0 & =q \omega+\frac{a}{2 \pi} \int_{0}^{q} \sin 2 \pi\left(\frac{p}{q} \theta+g_{0}^{\prime}-k \sin 2 \pi \theta\right) \mathrm{d} \theta=q \omega+\frac{a}{2 \pi} \sin 2 \pi g_{0}^{\prime} \int_{0}^{q} \cos 2 \pi\left(\frac{p}{q} \theta-k \sin 2 \pi \theta\right) \mathrm{d} \theta \\
& =q \omega+\frac{a}{2 \pi} \sin 2 \pi g_{0}^{\prime} \sum_{m=0}^{q-1} \int_{0}^{1} \cos 2 \pi\left(\frac{p m}{q}+\frac{p}{q} \theta-k \sin 2 \pi \theta\right) \mathrm{d} \theta \\
& =q \omega+\frac{a}{2 \pi} \sin 2 \pi g_{0}^{\prime}\left(\sum_{0}^{q-1} \cos 2 \pi \frac{p m}{q}\right) \int_{0}^{1} \cos 2 \pi\left(\frac{p}{q} \theta-k \sin 2 \pi \theta\right) \mathrm{d} \theta .
\end{aligned}
$$


After this somewhat exhausting exercise we note that the bracketed sum is zero unless $p / q \in \mathbb{Z}$. Thus, the cusp-like (high order contact) of the mode-locked regions observed for $\mathcal{L}_{(0,1 / 2)}$ also occurs here unless the resonance is of the form $(n, 0)$ for some $n \in \mathbb{Z}$, i.e., the boundary of the tongue is of the form

$$
\left|\Omega-\frac{p}{q} \alpha\right| \sim \mathrm{O}\left(A^{2}\right) \quad \text { if } \frac{p}{q} \notin \mathbb{Z} .
$$

On the other hand, if $p / q=n$ for some $n \in \mathbb{Z}$ (i.e., $q=1, p=n$ ) then (4.11) becomes

$$
0=\omega+\frac{a}{2 \pi} \sin 2 \pi g_{0}^{\prime} \int_{0}^{1} \cos 2 \pi(n \theta-k \sin 2 \pi \theta) \mathrm{d} \theta=\omega+\frac{a}{2 \pi} \sin 2 \pi g_{0}^{\prime} J_{n}(2 \pi k)
$$

using (A.5). Recalling the definition of $k$ the boundary of this mode-locked region may therefore be written as

$$
|\Omega-n \alpha| \sim \frac{A}{2 \pi}\left|J_{n}\left(\frac{\pi B}{|\sin \pi \alpha|}\right)\right| .
$$

Note, of course, that we could have taken $n \alpha(\bmod 1)$ and obtained the same result. Indeed, the different scalings predicted by this analysis presumably explains why the majority of mode-locked regions observed in [6] have rotation numbers of the form $n \alpha-[n \alpha]$ (where $[x]$ denotes the integer part of $x$ ). Moreover, by (A.8), at relatively large $B(4.14)$ becomes

$$
|\Omega-n \alpha| \sim \frac{A}{2 \pi^{2}}\left(\frac{2|\sin \pi \alpha|}{B}\right)^{1 / 2}\left|\cos \left(\frac{\pi B}{|\sin \pi \alpha|}-\frac{1}{2} n \pi-\frac{1}{4} \pi\right)\right| .
$$

\section{Numerical results}

The aim of this section is two-fold. First, we check the theoretical predictions described above for the mode-locked regions. Second, we look at how the mode-locked regions evolve outside the strict region of validity of the asymptotic results.

The computations of Figs. 2 and 3 have shown that for small $A$ and moderate $B$ the agreement between the theoretically predicted form of the mode-locked regions and the numerically calculated boundary is very good. In Fig. 4, we present similar calculations extended to a much larger range of the amplitude of the external forcing $B$. One can see that the theoretical prediction for $\mathcal{L}_{(0,0)}, \Omega \sim B^{-1 / 2}$, is reasonably satisfied when $A=0.1$, although violations from this behaviour start already at $B \approx 20$ if $A=0.2$. This emphasises the statement made at the beginning of Section 2 that the asymptotic analysis is valid for $A$ small and $B$ of order 1 . At larger values of the non-linearity $(A)$ and forcing there are two effects which are different to those expected from the asymptotic analysis. First, the pinching at moderate $B$ is much less marked than it is in the very small $A$ cases, where the width of the mode-locked region in the $\Omega$-direction very nearly vanishes. This is not surprising, since second-order effects, ignored in the analysis above, become important. Second, there appear to be intervals of $B$ values over which the width of the tongue vanishes. Both features are clearly seen in Fig. 1, where $A=0.8$, so we have chosen this parameter value for a more detailed investigation. The tongues were numerically determined as regions of a given constant rotation number. The latter was calculated using (1.5) via straightforward iterations of system (1.4). The only trick was to choose $n$ in such a way that $\theta_{n}$ is as close as possible to $\theta_{0}$ (in other words, we took the best recurrences of the driving map). For the golden mean $\alpha$ this corresponds simply to the choice $n=F_{k}$, where $F_{k}$ is a Fibonacci number. This choice essentially improves the accuracy compared to a randomly chosen $n\left(1 / n^{2}\right.$ compared to $1 / n$ ). The boundary of the tongue was then located at a value of $\Omega$ (for fixed $B$ ), where the rotation value sufficiently deviates from the given rational. The region $B \approx 45$ in Fig. $4 \mathrm{~b}$ below, where the width of the tongue appears to be zero, gives an impression on the accuracy of the method. 

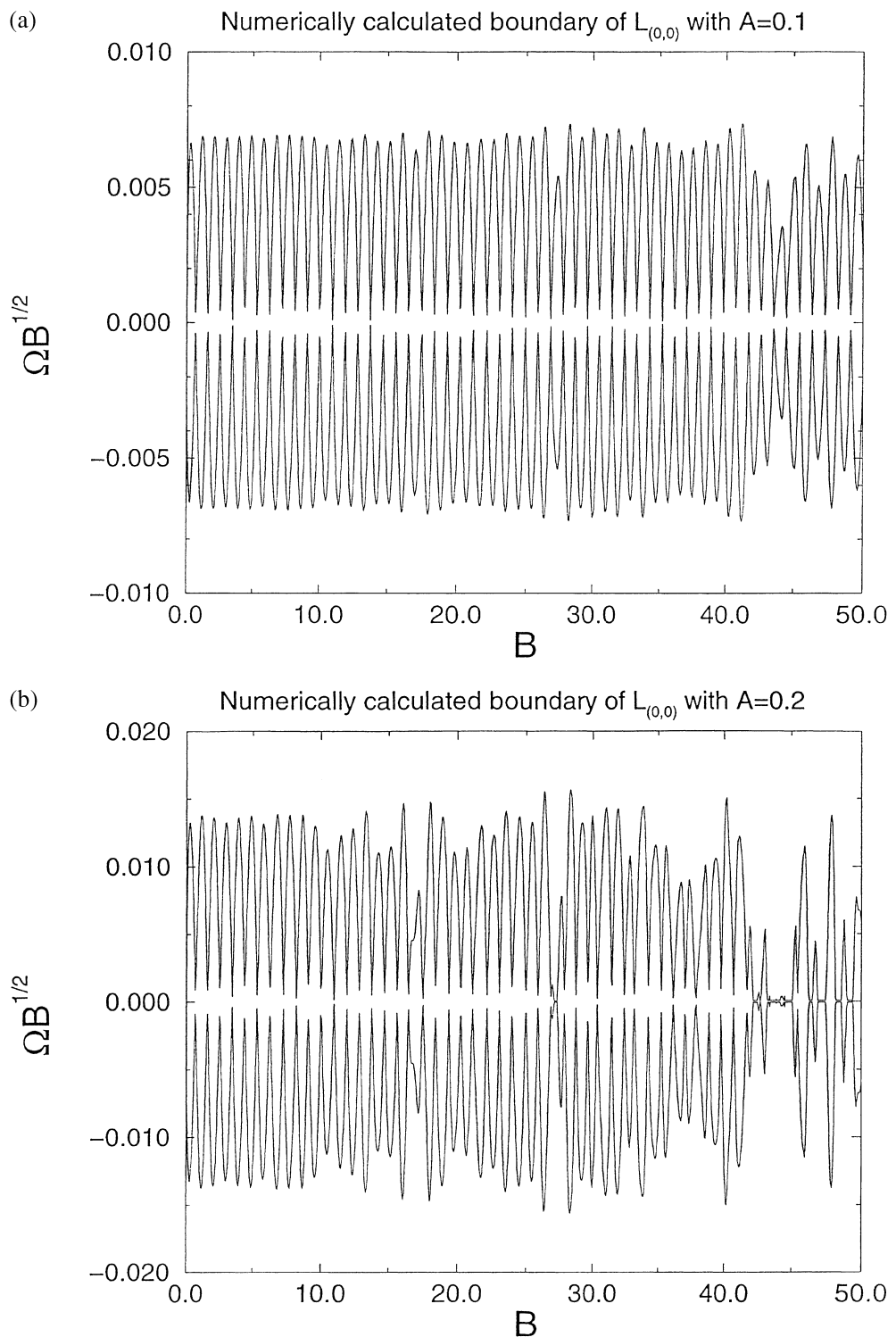

Fig. 4. The boundary of $\mathcal{L}_{(0,0)}$ for (1.4) with $\alpha=\frac{1}{2}(\sqrt{5}-1)$ over a large range of $B$ : (a) $A=0.1$; (b) $A=0.2$.

Fig. 5 shows the bifurcation diagram at the pinching point near $B=0.7$ (an enlargement of the structure inside the dashed square of Fig. 1). To get a rough picture of the bifurcation structure in Fig. 5 we have computed the saddle-node bifurcations by checking the transition from mono- to bistability crossing the bifurcation line under the variation of $B$ with fixed $\Omega$. This transition can be monitored by iterating a large number of initial conditions on a line in phase space, where the values for $x$ are equally distributed in the interval $[0,1]$ and $\theta$ is fixed. In the case of monostability all these initial conditions collapse to one point after some transients, while after the bifurcation we obtain more than one point. This picture was the basis for more accurate computations of the saddle-node bifurcation as the collision point of the stable and unstable invariant curves computed from forward and backward iteration. 


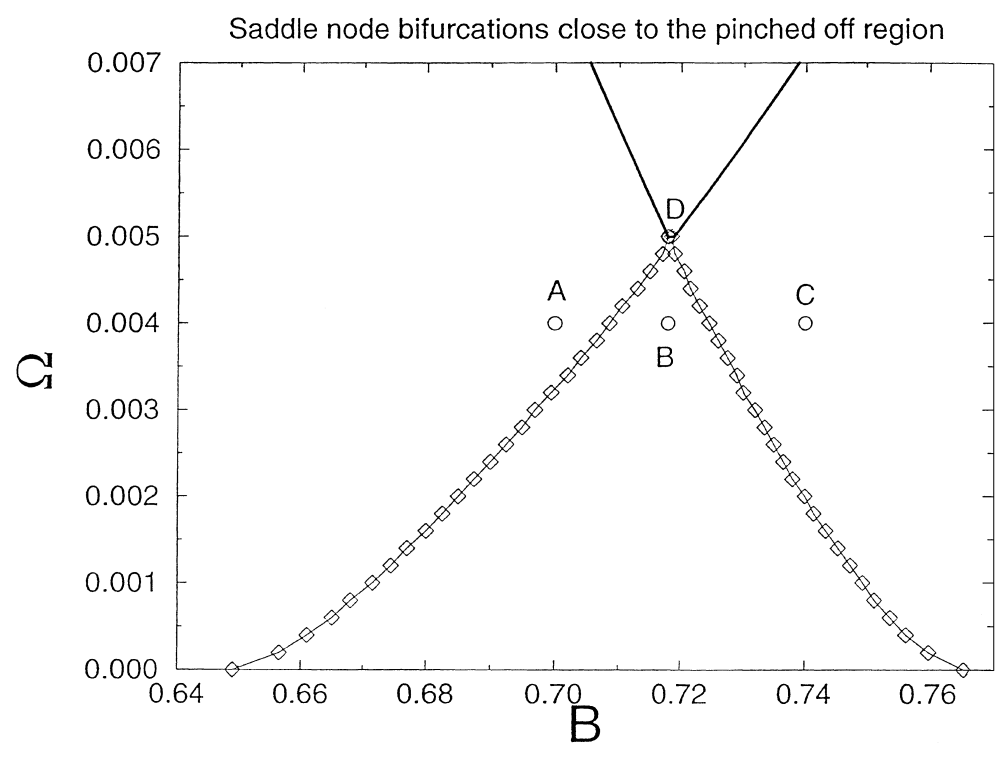

Fig. 5. Enlargement of the rectangle in Fig. 1 showing that the tongue does not actually pinch off completely; two curves of saddle-node bifurcations (of invariant curves) cross.

There are three regions with qualitatively different dynamics. As shown in Fig. 6a and c, in regions $A$ and $C$ there is a single attracting closed invariant curve for the map (1.4), and an unstable invariant curve (not shown). On crossing the bifurcation line marked in diamonds a simple saddle-node bifurcation of invariant curves produces an additional pair of invariant curves, one stable and the other unstable, so in region $B$ (see Fig. 6b) there are two stable invariant curves. Thus, there are two curves of saddle-node bifurcations of invariant curves, which intersect at a bi-critical point. The dynamics of the quasi-periodic motion just above the bi-critical point $\mathrm{D}$ is illustrated in Fig. 6d: intermittent behaviour associated with both of the stable invariant curves is clearly seen.

Another feature which influences the bifurcation diagram at larger non-linearity and forcing amplitudes is the appearance of strange non-chaotic attractors. For a general discussion of such attractors see $[3,9,14]$ and in the context of quasi-periodically forced circle maps see [5-7]. A strange non-chaotic attractor is associated with the apparent vanishing of the width of the mode-locked tongues at larger values of $B$ ( $B \sim 3$ in Fig. 1). This transition has been described in [6,7]; the strange non-chaotic attractor appears through a non-smooth saddle-node bifurcation: the stable and unstable invariant curves do not coincide at the bifurcation, but appear to touch on a set of points whose projection onto the $\theta$ axis is dense. As has been argued in [7], the appearance of the strange non-chaotic attractor with $\rho=0$ is very sensitive to perturbations, and as a result $\rho$ appears to vary continuously as $\Omega$ passes through zero, leading to a mode-locked region of zero (or very small) width.

Putting this information together, the multiple saddle-node bifurcations and intervals on which the width of the tongue effectively vanishes, we obtain a global picture of the $\rho=0$ mode-locked tongue as shown in Fig. 7. Cutting through this three-dimensional structure at constant small $A$ shows a number of bubbles (isolas) of saddle-node bifurcations of invariant curves with small overlap regions between successive bubbles (as shown in Fig. 5). These overlaps would presumably appear in the theory if the perturbation analysis above were pushed through to higher order. As $A$ is increased, some of these bubbles are pulled apart, and intervals of (apparently) zero width for the tongues appear between the successive bubbles. Note that since the rotation number is continuous, the tongue can never vanish completely, and in an appropriate two-parameter projection the mode-locked region is represented by a curve between the bubbles if the width really does go to zero. Fig. 7 is highly speculative and certainly incomplete 
(a)

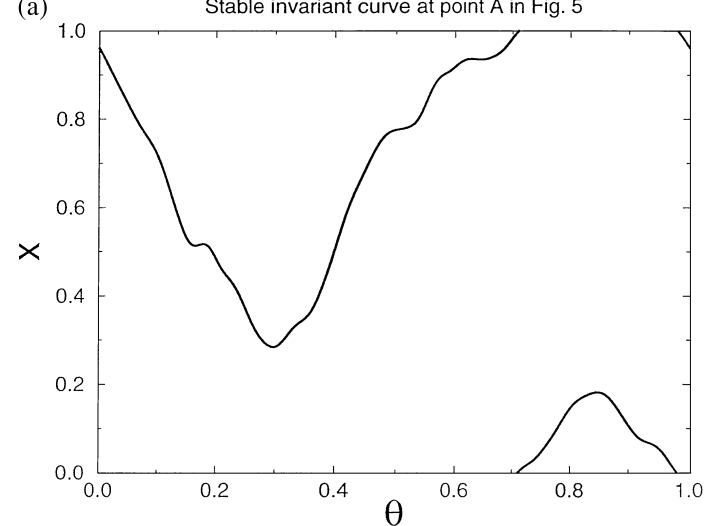

(c)

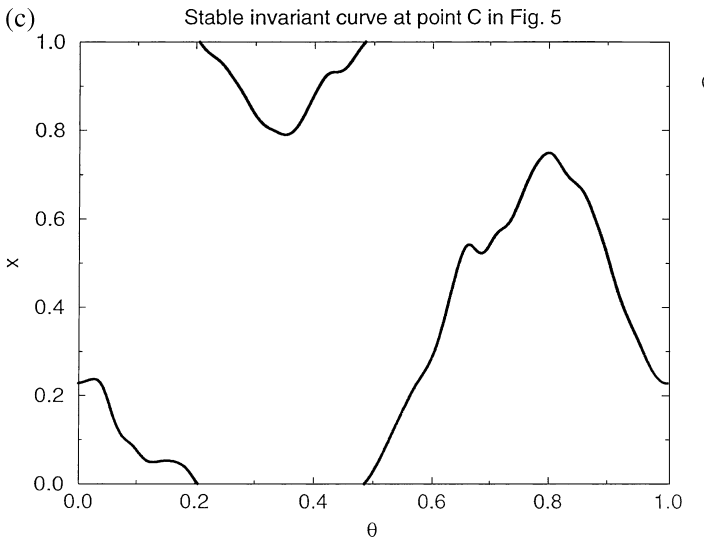

(b)

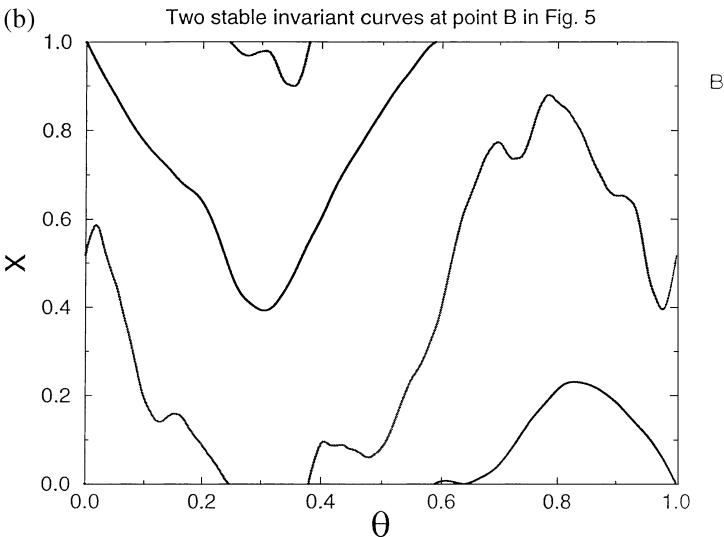

(d) Quasiperiodic motion outside the mode-locked region at point D in Fig. 5

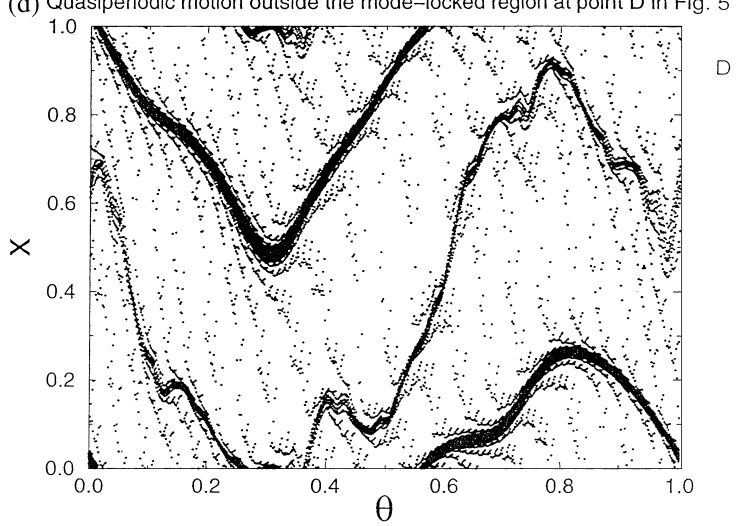

Fig. 6. Phase portraits showing the dynamics in each of the four regions $A, B, C$ and $D$ of Fig. 5. (a) The unique stable invariant curve at $A$; (b) a pair of stable invariant curves at $B ;$ (c) the unique stable invariant curve at $B$; and (d) an orbit at $D$ showing intermittency near the locus both invariant curves which exist at nearby parameters.

(there must be some additional saddle-node bifurcations), but it fits the theoretical and numerical results of which we are aware. The parameter values at which the bubbles first separate is a natural focus for further work on the appearance of strange non-chaotic attractors, and we should emphasise that it is not clear whether the width of the tongues can become zero, or whether the width is just very small.

\section{General circle maps}

The asymptotic analysis of Section 2 may be repeated for general maps of the form

$$
x_{n+1}=x_{n}+\Omega+\epsilon F\left(x_{n}\right)+B H\left(\theta_{n}\right), \quad \theta_{n+1}=\theta_{n}+\alpha,
$$

where $F$ and $H$ are smooth periodic functions of period $1,0<\epsilon \ll 1, B$ is a positive real parameter and $\alpha$ is irrational. By redefining $\Omega$ we may assume without loss of generality that both $F$ and $H$ have zero mean. The mode-locked region $\mathcal{L}_{(0,0)}$ can be found to lowest order by posing $\Omega \sim \omega \epsilon$ and looking for an invariant curve of the form $G_{0}(\theta)+\epsilon G_{1}(\theta)$, where $G_{0}$ and $G_{1}$ are periodic of period 1 . In this case we obtain

$$
G_{0}(\theta+\alpha)=G_{0}(\theta)+B H(\theta),
$$




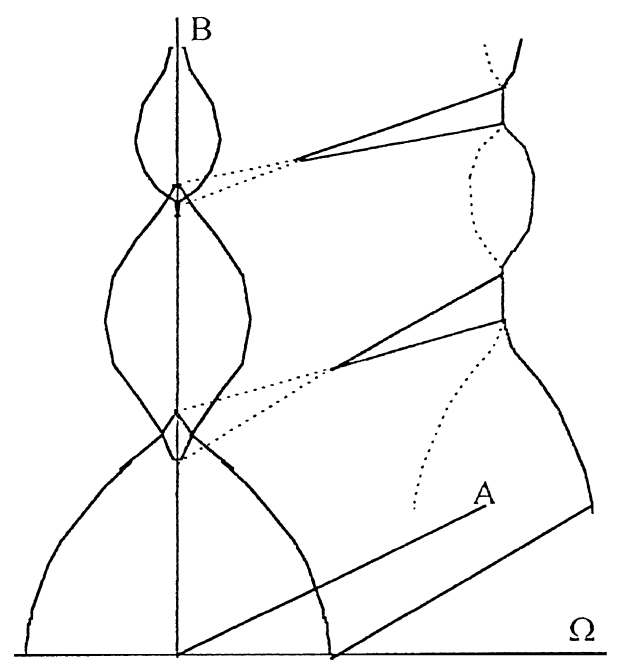

Fig. 7. A schematic view of the conjectured structure of the mode-locked region in three-dimensional parameter space showing how the 'bubbles' of saddle-node bifurcations of invariant curves might separate at larger values of $A$, producing sheet-like regions of the mode-locked tongues.

and

$$
G_{1}(\theta+\alpha)=G_{1}(\theta)+\omega+F\left(G_{0}(\theta)\right)
$$

The lowest order problem, (6.2), may be formally solved as a series solution

$$
G_{0}(\theta)=g_{0}+B \sum_{m=1}^{\infty} H(\theta-m \alpha)
$$

where $g_{0}$ is an arbitrary parameter. So, at least formally, $G_{0}(\theta)=g_{0}+B h(\theta)$ where $h$ is a period 1 , zero mean function. The consistency condition is thus (integrating (6.3) from 0 to 1 )

$$
-\omega=\int_{0}^{1} F\left(g_{0}+B h(\theta)\right) .
$$

Since $F$ is periodic of period 1, we may expand it as a Fourier series

$$
F(\theta)=\sum_{k \geq 1}\left(A_{k} \sin (2 \pi k \theta)+B_{k} \cos (2 \pi k \theta)\right),
$$

and so the integral on the right-hand side of (6.5) may be rewritten, assuming that the order of the sum and integral can be exchanged, as

$$
\sum_{k \geq 1}\left(A_{k} \int_{0}^{1} \sin \left(2 \pi k B\left(h(\theta)+B^{-1} g_{0}\right)\right) \mathrm{d} \theta+B_{k} \int_{0}^{1} \cos \left(2 \pi k B\left(h(\theta)+B^{-1} g_{0}\right)\right) \mathrm{d} \theta\right) .
$$

The method of stationary phase can be applied to each of the integrals if $B$ is large:

$$
\int \cos 2 \pi B \psi(\theta) \mathrm{d} \theta \sim B^{-1 / 2} \sum_{m} \frac{1}{\sqrt{\psi^{\prime \prime}\left(\theta_{m}\right)}} \cos 2 \pi\left(B \psi\left(\theta_{m}\right)+\frac{1}{8} \operatorname{sign}\left(\psi^{\prime \prime}\left(\theta_{m}\right)\right)\right),
$$


where the sum is over the saddle points of $\psi$ within the range of integration. Assuming again that the order of summations may be exchanged, then (6.8) applied to the expression of (6.7) gives

$$
B^{-1 / 2} \sum_{m} \frac{1}{\sqrt{\left|h^{\prime \prime}\left(\theta_{m}\right)\right|}} \sum_{k \geq 1} \mathcal{S}_{k}\left(h\left(\theta_{m}\right) B+g_{0}+\frac{1}{8} \sigma_{m}\right),
$$

where

$$
\mathcal{S}_{k}(z)=\frac{A_{k}}{\sqrt{k}} \sin 2 \pi k z+\frac{B_{k}}{\sqrt{k}} \cos 2 \pi k z
$$

and the sum over $m$ is of all the saddle points, $\theta_{m}$, of the function $h$ in $[0,1]$ and $\sigma_{m}=\operatorname{sign}\left(h^{\prime \prime}\left(\theta_{m}\right)\right)$. For each $m$, the sum over $k$ in (6.9a) and (6.9b) defines the Fourier series of a perfectly well-behaved periodic function with period 1 , which is at least as smooth as $F$ (it is the function with $k$ th Fourier coefficient equal to the Fourier coefficient of $F$ divided by $\sqrt{k}$, i.e., $D^{-1 / 2} F(\theta)$, where $D$ is the usual differential operator). Let this function be $\mathcal{F}(x)$, i.e.,

$$
\mathcal{F}(x)=\sum_{k \geq 1} \mathcal{S}_{k}(x)
$$

then if $B$ is large (but small compared to $\epsilon^{-1}$ ), then (6.5) is

$$
0 \sim \omega+B^{-1 / 2} \sum_{m} \alpha_{m} \mathcal{F}\left(h_{m} B+\frac{1}{8} \sigma_{m}+g_{0}\right)
$$

where $\alpha_{m}=1 / \sqrt{\left|h^{\prime \prime}\left(\theta_{m}\right)\right|}$ and $h_{m}=h\left(\theta_{m}\right)$.

Pinching occurs at $B=B^{*}$ such that

$$
\sum_{m} \alpha_{m} \mathcal{F}\left(h_{m} B^{*}+\frac{1}{8} \sigma_{m}+g_{0}\right)=0 \text { for all } g_{0} .
$$

For general functions $H$ and $F$ (and hence $\mathcal{F}$ ) there is no obvious reason why the pinching condition (6.12) should hold for any $B^{*}$, and so we expect that in general the pinching effect should not occur. In fact, we shall show that pinching occurs due to some of the symmetries of the quasi-periodically forced circle maps generally considered.

Suppose that $H$ and so by (6.4) $h$ is odd possibly after a rotation of coordinates. Then (and this is the important condition) if $\theta_{m}$ is a stationary point, there is unique stationary point in $[0,1], \theta_{-m}$ say, such that

$$
h_{-m}=-h_{m}, \quad \alpha_{-m}=\alpha_{m}, \quad \sigma_{-m}=-\sigma_{m} .
$$

Thus (6.9a) and (6.9b) can be written as a sum over $|m|$ :

$$
B^{-1 / 2} \sum_{|m|} \alpha_{|m|} \sum_{k \geq 1} \mathcal{S}_{k}\left(h_{|m|} B+\frac{1}{8} \sigma_{|m|}+g_{0}\right)+\mathcal{S}_{k}\left(-h_{|m|} B-\frac{1}{8} \sigma_{|m|}+g_{0}\right) .
$$

Using (6.9b) the sum of $\mathcal{S}_{k}$ s can be simplified, giving

$$
\begin{gathered}
2 B^{-1 / 2} \sum_{\{m \mid m>0\}} \alpha_{m} \sin 2 \pi g_{0}\left[\sum_{k \geq 1} \frac{A_{k}}{\sqrt{k}} \cos 2 \pi k\left(h_{m} B+\frac{1}{8} \sigma_{m}\right)\right] \\
+2 B^{-1 / 2} \sum_{\{m \mid m>0\}} \alpha_{m} \cos 2 \pi g_{0}\left[\sum_{k \geq 1} \frac{B_{k}}{\sqrt{k}} \cos 2 \pi k\left(h_{m} B+\frac{1}{8} \sigma_{m}\right)\right] .
\end{gathered}
$$


Denoting the two functions defined by the Fourier sums in square brackets by $P(z)$ and $Q(z)$, respectively (for $\left.z=h_{m} B+\frac{1}{8} \sigma_{m}\right)$, the consistency condition (6.5) is thus

$$
-B^{1 / 2} \omega=\sin 2 \pi g_{0}\left[\sum_{\{m \mid m>0\}} P\left(h_{m} B+\frac{1}{8} \sigma_{m}\right)\right]+\cos 2 \pi g_{0}\left[\sum_{\{m \mid m>0\}} Q\left(h_{m} B+\frac{1}{8} \sigma_{m}\right)\right] .
$$

Thus, we see immediately that if either $P(z)=0$ for all $z$ or $Q(z)=0$ for all $z$, i.e., if either $A_{k}=0$ for all $k \geq 1$ or $B_{k}=0$ for all $k \geq 0$, then we obtain a separation of the variables and, e.g., if $Q(z)$ is identically zero, the lowest order (large $B$ ) condition for made-locking is

$$
|\omega| \leq 2 B^{-1 / 2}\left|\sum_{\{m \mid m>0\}} P\left(h_{m} B+\frac{1}{8} \sigma_{m}\right)\right| .
$$

The right-hand side of this equation is independent of $g_{0}$ as it should be, and there is pinching provided that there exists $B^{*}$ such that

$$
\sum_{\{m \mid m>0\}} P\left(h_{m} B^{*}+\frac{1}{8} \sigma_{m}\right)=0 .
$$

Since $P$ is a periodic zero mean function, then (6.17) must be satisfied infinitely often.

It is worth emphasising that it is only the symmetry of $F$ and condition (6.13) on the coefficients associated with the stationary points of $h$ that are required for this argument to hold. It does show that the pinching observed both numerically and in physical experiments is a feature of the symmetries of the problem.

For the standard example considered above, $F(x)=(A / 2 \pi) \sin 2 \pi x$ and $H(x)=\sin 2 \pi \theta$ with

$$
h(\theta)=-\frac{1}{2 \sin \pi \alpha} \sin 2 \pi(\theta-\phi)
$$

with $\phi=\frac{1}{2} \alpha-\frac{1}{4}$. This has a (symmetric) pair of stationary points and we may take $\theta_{1}=\frac{1}{2} \alpha$ with $h_{1}=h\left(\theta_{1}\right)=$ $-1 /(2 \sin \pi \alpha)$ and $h^{\prime \prime}\left(\theta_{1}\right)=-4 \pi^{2} h_{1}$ so that $\sigma_{1}=-\operatorname{sign} h_{1}$. Substituting into (6.11) with $A_{1}=A / 2 \pi$ and all the other Fourier coefficients equal to zero, we obtain

$$
\frac{1}{\pi}\left(\frac{2|\sin \pi \alpha|}{B}\right)^{1 / 2} \cos 2 \pi\left(-\frac{B}{2 \sin \pi \alpha}+\frac{1}{8}+\frac{1}{8} \operatorname{sign}(\sin \pi \alpha)\right)
$$

which after a little more thought, is equivalent to (2.11).

We should emphasise that this analysis is not, strictly speaking, valid. In the perturbation expansions to obtain (6.2) and (6.3) we have assumed that $B$ is of order 1 (otherwise the desired splitting of the different orders does not hold). On the other hand, in order to apply the method of stationary phase we have assumed that $B$ is large. Thus, our assumptions are only valid if we can choose $B$ to be order 1 and large enough for the method of stationary phase to give reasonable results. This is clearly unreasonable, but we feel that the above analysis, flawed though it is, still provides an explanation for the observed phenomena and might be made more rigourous if sensible scalings for large $B$ and small $A$ could be found. This caveat does not apply to the analysis of earlier sections because it was not necessary to assume that $B$ is large there - it is relevant, of course, to the asymptotic expressions of (2.11) and (3.11) in which large $B$ expansions of the Bessel functions are used. 


\section{Conclusion}

Using perturbation theory we have given a fairly complete description of the dominant mode-locked regions for a quasi-periodically forced circle map in the limit of small non-linearity. These regions have a relatively complicated structure compared to the equivalent regions in simple circle maps. Our main motivation for this work was the understanding of the appearance of strange non-chaotic attractors in such maps. Although this paper does not address this interesting problem explicitly we believe that these results should help orient research into the structure of such attractors in parameter space.

Note. The conjecture in Section 5 about higher order perturbation theory and the overlap of the tongues has been confirmed: see Ref. [18].

\section{Acknowledgements}

We are grateful for the support of the British Council and DAAD, and to an EU network grant on spatially extended dynamical systems which enabled us to work together. UF acknowledges support from the Deutsche Forschungsgemeinschaft.

\section{Appendix A}

For the evaluation of various integrals which arise in this paper we have relied on Abramowitz and Stegun [1, Chapter 9]. In this appendix the main results used are collected together for the readers convenience.

For $n$ a non-negative integer

$$
\frac{1}{\pi} \int_{0}^{\pi} \cos (n \theta-z \sin \theta) \mathrm{d} \theta=J_{n}(z),
$$

where $J_{n}$ is the Bessel function of order $n$. Also

$$
J_{-n}(z)=(-1)^{n} J_{n}(z),
$$

so that

$$
J_{n}(z)=J_{-n}(-z)=(-1)^{n} J_{n}(-z),
$$

and

$$
\left|J_{|n|}(|z|)\right|=\left|J_{n}( \pm z)\right|=\left|J_{ \pm n}(z)\right| .
$$

Our normalization is a little different, but from (A.1) it is easy to see that

$$
\int_{0}^{1} \cos 2 \pi(n \theta-z \sin 2 \pi \theta) \mathrm{d} \theta=J_{n}(2 \pi z)
$$

and by symmetry arguments

$$
\int_{0}^{1} \sin 2 \pi(n \theta-z \sin 2 \pi \theta) \mathrm{d} \theta=0 .
$$

Thus, if $C, k$ and $\phi$ are real constants and $n$ is a non-negative integer, 


$$
\begin{aligned}
\int_{0}^{1} \sin 2 \pi(C+n \theta-k \sin 2 \pi(\theta-\phi)) \mathrm{d} \theta & =\int_{0}^{1} \sin 2 \pi(C+n \phi+n \theta-k \sin 2 \pi \theta) \mathrm{d} \theta \\
& =\sin 2 \pi(C+n \phi) \int_{0}^{1} \cos 2 \pi(n \theta-k \sin 2 \pi \theta) \mathrm{d} \theta \\
& =\sin 2 \pi(C+n \phi) J_{n}(2 \pi k) .
\end{aligned}
$$

Note that in this calculation we have used the fact that if $f$ is integrable and of period 1 then $\int_{0}^{1} f(\theta) \mathrm{d} \theta=$ $\int_{0}^{1} f(\theta-\phi) \mathrm{d} \theta$ for any real constant $\phi$. Finally, as $z \rightarrow \infty$,

$$
J_{n}(z) \sim\left(\frac{2}{\pi z}\right)^{1 / 2} \cos \left(z-\frac{1}{2} n \pi-\frac{1}{4} \pi\right)+\mathrm{O}\left(\frac{1}{z}\right) .
$$

\section{References}

[1] M. Abramowitz, I.A. Stegun, Handbook of Mathematical Functions, Dover, New York, 1965.

[2] D.K. Arrowsmith, C.M. Place, An Introduction to Dynamical Systems, CUP, Cambridge, 1992.

[3] A. Bondeson, E. Ott, T.M. Antonsen Jr., Phys. Rev. Lett. 55 (1985) 2103.

[4] P.R. Chastell, P.A. Glendinning, J. Stark, Phys. Lett. A 200 (1995) 17.

[5] M. Ding, C. Grebogi, E. Ott, Phys. Rev. A 39 (1989) 2593.

[6] U. Feudel, C. Grebogi, E. Ott, Phys. Rep. 290 (1997) 11.

[7] U. Feudel, J. Kurths, A.S. Pikovsky, Physica D 88 (1995) 176.

[8] P. Glendinning, Phys. Lett. A. 244 (1998) 545.

[9] C. Grebogi, E. Ott, S. Pelikan, J.A. Yorke, Physica D 13 (1984) 261.

[10] J.F. Heagy, S.M. Hammel, Physica D 70 (1994) 140.

[11] M.R. Herman, Comment. Math. Helvetici 58 (1983) 453.

[12] S.P. Kuznetsov, A.S. Pikovsky, U. Feudel, Phys. Rev. E 51 (1995) R1629.

[13] T. Nishikawa, K. Kaneko, Phys. Rev. E 54 (1996) 6114.

[14] A. Pikovsky, U. Feudel, Chaos 5 (1995) 253.

[15] A. Prasad, V. Mehra, R. Ramaswamy, Phys. Rev. Lett. 79 (1997) 4127.

[16] F.J. Romeiras, A. Bondeson, E. Ott, T.M. Anderson Jr., C. Grebogi, Phys. Scr. 40 (1989) 442.

[17] T. Yalcinkaya, Y.-C. Lai, Phys. Rev. Lett. 77 (1996) 5039.

[18] P. Glendinning, J. Weirsig, Phys. Lett. A 257 (1999) 65-69. 